\title{
EL CICLOTRÓN Y LA FACULTAD DE CIENCIAS DE LA UNIVERSIDAD DE CHILE
}

\author{
José Roberto Morales Peña
}




\section{JOSÉ ROBERTO MORALES PEÑA}

Es Profesor de Estado en Matemáticas y Física y Doctor en Física por la Universidad de California, Davis. Es Profesor Titular de la Universidad de Chile y Director del Centro de Física Experimental de la Facultad de Ciencias del mismo plantel. 


\section{EL CICLOTRÓN Y LA FACULTAD DE CIENCIAS DE LA UNIVERSIDAD DE CHILE}

Han pasado 50 años desde que el Decreto Universitario $\mathrm{N}^{\circ} 135$ del 14 de enero de 1965 creara la Facultad de Ciencias de la Universidad de Chile. Su creación vino a reconocer el interés de un importante grupo de académicos motivados por investigar las fronteras de la ciencia y formar jóvenes investigadores en un ambiente propio e independiente. En estos 50 años los objetivos originales se han cumplido y es hoy una unidad académica con reconocimiento nacional e internacional. Al presente la facultad ocupa parte del Campus Juan Gómez Millas, el que comparte con otras facultades. Hace 50 años ese terreno, que era de uso agrícola, le fue cedido a la Universidad de Chile para que en él se instalara la naciente facultad. ¿Por qué se eligió este lugar y no otro? Una buena razón tuvo que ver con que era el más adecuado para instalar ahí un acelerador de partículas, el ciclotrón1, un complejo aparato para investigar los núcleos atómicos. El ciclotrón pasó a convertirse en un símbolo de la facultad y posteriormente del campus. En lo que sigue recordaremos cómo se gestó la incorporación del ciclotrón al patrimonio de la Universidad y sus aportes a la actividad académica.

\section{ASPECTOS HISTÓRICOS DE RELEVANCIA}

Que la Universidad de Chile contara con un acelerador de partículas para investigar en física nuclear no era algo novedoso, ya que en la Escuela de Ingeniería, en el Departamento de Física, había un grupo de físicos nucleares, varios con doctorado, que en sus investigaciones utilizaban un acelerador de baja energía, $800 \mathrm{keV}$ máxima, de tipo Cockcroft-Walton y adquirido a la firma Phillips de Holanda cerca de 1955. Después de varios años, en el grupo había interés en adquirir un acelerador de mayor energía para abordar problemas más interesantes en física nuclear. Los esfuerzos encabezados por el Dr. Jacobo Rapaport para adquirir un nuevo acelerador, de tipo Van de Graaff, fracasaron pese a haberse negociado un préstamos favorable, por lo que él decidió seguir su carrera científica en EE.UU.

La creación de la Facultad de Ciencias contó desde el comienzo con el apoyo de la Universidad de California, EE.UU. dentro del marco de un convenio2 entre esa universidad y la nuestra. El convenio auspiciaba actividades como visitas de directivos de ambas universidades, pasantías académicas, donación de equipos y 
becas otorgadas por la Ford Foundation a jóvenes académicos chilenos para cursar estudios de magíster y doctorado en las distintas sedes de la U. de California.

En su paso por el campus de Davis, en el marco de una visita de autoridades de la Universidad de Chile a los distintos campus de la Universidad de California, se reunieron autoridades y académicos para explorar posibilidades de intercambio. La misión chilena estaba encabezada por el Prorrector y el Decano de la Facultad de Agricultura. Entre los académicos norteamericanos estaban el Dr. John Jungerman3, director del Crocker Nuclear Laboratory, situado en el campus de la ciudad de Davis, y el Dr. William Knox, ambos físicos nucleares y profesores en el Departamento de Física de UC Davis. De acuerdo a lo relatado por el Dr. Jungerman fue en una de estas reuniones que el Profesor William Knox mencionó que había en ese campus un acelerador de tipo ciclotrón de baja energía, que había quedado sin uso porque estaban construyendo uno de mayor energía y que tal vez ese acelerador podría interesar a los físicos de la U. de Chile. Entre los visitantes chilenos no había físicos, pero la posibilidad de contar con un acelerador les pareció interesante y la comunicaron rápidamente al Departamento de Física de la Escuela de Ingeniería.

La oferta de un acelerador ciclotrón de "segunda mano" abría la posibilidad de una renovación de equipos a bajo costo. El Profesor Jorge Zamudio se interesó en el ciclotrón por la posibilidad de poder realizar investigaciones en física de neutrones a energías mayores que las que podían lograrse con el Cockcroft-Walton. El Dr. Zamudio había retornado de Francia después de finalizar su doctorado en física nuclear y había montado un experimento con neutrones en el acelerador CrockoftWalton. Con el ciclotrón de UC Davis podría logarse neutrones con energías mayores, del orden de $20 \mathrm{MeV}$, lo que permitiría estudiar reacciones nucleares en un rango de energía poco explorado.

\section{EL CICLOTRÓN DE 22”}

El acelerador ofrecido a la Universidad de Chile tiene una historia muy interesante que resumimos a continuación. En efecto, se construyó en la Universidad de California por un grupo de físicos de UC Davis interesados en estudiar decaimientos beta. Para un experimento requirieron de un haz de partículas cargadas y pensaron que un pequeño acelerador de tipo Van de Graaff podría servirles. Por lo tanto, fueron a UC Berkeley, distante unos $100 \mathrm{~km}$, a ver si podrían armar uno usando las numerosas partes de equipos ya usados en diferentes proyectos. El Profesor E. Lawrence4 les dijo que dispusieran de lo que quisieran, pero que armaran un ciclotrón y no un Van de Graaff. Así, se gestó la construcción del ciclotrón de 22", que después vino a Chile, usando un electroimán de veintidós pulgadas de diámetro. El poderoso el 
campo magnético del ciclotrón afectaba las trayectorias de partículas beta en un experimento de otro grupo, por lo que hubo que encerrar al ciclotrón en una caja de paredes de hierro de una pulgada de espesor y dimensiones de 3,8 $\mathrm{m}$ x 3,9 $\mathrm{m}$ x 2,3 m. Este acelerador estuvo en operación en UC Davis hasta 1964, cuando se puso en operación otro ciclotrón de mayor tamaño y con capacidad para generar haces de iones con energías cercanas a $100 \mathrm{MeV}$.

\section{LA TRANSFERENCIA DEL CICLOTRÓN A LA UNIVERSIDAD DE CHILE}

La decisión de aceptar el ofrecimiento de donar a la U. de Chile el ciclotrón de UC Davis y traerlo al país implicó afrontar numerosos problemas de diversa índole, desde lo administrativo a lo económico, pasando por lo académico. El Dr. Zamudio lideró el grupo encargado de resolverlos. La Fundación Ford hizo un importante aporte económico que permitió complementar la donación con algunos equipos anexos. Un problema no menor era que la instalación del ciclotrón requería de un edificio y de un espacio que no estaban disponibles en la Escuela de Ingeniería, por lo que hubo que buscar un terreno para este fin. Por otra parte, el ciclotrón pasó a depender administrativa y académicamente de la naciente Facultad de Ciencias, pero esta carecía de una sede propia y tenía a sus investigadores dispersos en distintas facultades. Los esfuerzos para conseguir un terreno adecuado que acogiera tanto al ciclotrón como a la Facultad de Ciencias culminaron exitosamente con la cesión a la U. de Chile de un amplio terreno ubicado en un sector de la chacra Santa Julia, rodeado por la avenida Macul, la avenida Grecia, la calle Los Presidentes y calle Las Encinas. Hoy es el Campus Juan Gómez Millas.

El primer edificio que se construyó en ese terreno fue para el ciclotrón, que pasó a ser el símbolo de ese nuevo desarrollo académico de la Universidad de Chile.

Las obras se iniciaron en 1966 y lo primero que se construyó fue el radier sobre el cual se posó la caja de hierro que lo contenía. El resto del edificio, un galpón de grandes dimensiones, de aproximadamente $25 \mathrm{~m}$ de largo, $17,5 \mathrm{~m}$ de ancho y $9 \mathrm{~m}$ de alto, se fue construyendo en su entorno.

La llegada del ciclotrón y su instalación en Macul coincidió con el cese definitivo de experimentos con el acelerador Crockoft-Walton. El último trabajo fue en física de neutrones y se publicó en 19675.

La puesta en operación del ciclotrón demandó un trabajo técnico especializado para el cual se contó con la colaboración de ingenieros del Crocker Nuclear Laboratory de UC Davis y de técnicos chilenos. El primer haz se obtuvo en 1967. El edificio que lo albergó se diseñó amplio, siguiendo el modelo del que se construyó para el nuevo acelerador de UC Davis. Contemplaba tres áreas, una para 
ciclotrón, su blindaje de concreto, la unidad de radiofrecuencia y los rectificadores de silicio que cambiaban la tensión alterna de la línea de alimentación a tensión continua de 5 volts. La segunda sección quedó para trabajos con partículas cargadas, en la que se instaló una cámara especial, y la tercera sección se construyó para realizar investigaciones con neutrones. Esta última tenía paredes de concreto de aproximadamente un metro de espesor y era casi esférica, de aproximadamente $10 \mathrm{~m}$ de diámetro. La intención fue contar con un laboratorio capaz de apoyar la realización de trabajos de investigación en física nuclear de bajas energías utilizando haces de partículas cargadas (protones, deuterones y alfas) proporcionadas por el ciclotrón, con la posibilidad de variar las energías entre $2 \mathrm{MeV}$ y $10 \mathrm{MeV}$ y además, mediante una reacción nuclear especial6, producir neutrones de $20 \mathrm{MeV}$.

\section{ANÉCDOTAS}

La traída del ciclotrón a Chile, su instalación y la respectiva inauguración no estuvieron exentas de ciertos hechos anecdóticos que es interesante recordar. El primero ocurrió al desembarcar la caja metálica dentro de la cual estaba soldado el ciclotrón. Este había sido trasladado desde Davis hasta el puerto de San Francisco y embarcado en un barco mercante nacional que venía desde Japón con muy poca carga. Los ingenieros del barco decidieron, para mayor seguridad, bajar la caja hasta la bodega más profunda y encadenarla al barco. La tripulación se renovó en ese puerto y la información relativa al aseguramiento de la caja no fue traspasada a la nueva. Cuando el barco llegó a Valparaíso, al momento de retirar la caja se observó que esta no se levantaba pese al esfuerzo de las grúas del puerto. Finalmente, una revisión exhaustiva descubrió la cadena y el ciclotrón pudo ser removido del barco.

La segunda anécdota tiene que ver con el traslado desde el puerto hasta el predio asignado en Macul. El radier sobre el cual se posaría la caja había sido terminado a tiempo, pero se encontraba a unos cincuenta metros del portón de entrada en calle Las Palmeras, en medio de un terreno que hasta entonces era de uso agrícola. El Dr. Zamudio se había preocupado de reforzar con ripio el trayecto por el que maniobraría el camión y su acoplado. La noche anterior llovió y el suelo se reblandeció. El chofer encargado de traer este curioso cargamento se adelantó, llegó antes de lo previsto y decidió realizar la maniobra confiando en su experiencia. El resultado fue que al pisar el terreno blando, las ruedas delanteras del camión se hundieron y hubo que realizar una maniobra adicional para llevar la caja de hierro con el ciclotrón en su interior hasta el sitio correcto. La construcción del edificio se inició a continuación.

En años posteriores, durante la dictadura, el edificio del ciclotrón dio refugio a algunos atemorizados jóvenes que huían después de haber gritado contra el régimen 
en la avenida Grecia o en la avenida Macul. En una ocasión, unos diez de ellos se sentaron en torno a una mesa de trabajo escuchando atentamente la clase de física nuclear que el profesor improvisó para ellos. Ninguno de ellos era estudiante de física y esa clase habrá sido para ellos inolvidable. Al oficial que llegó a revisar el recinto se le hizo notar que estaba interrumpiendo una clase de posgrado.

En esa época en que los medios de expresión estaban controlados, un grupo de estudiantes armó un rudimentario emisor de radio que a veces hacía emisiones clandestinas. Los sistemas de seguridad no pudieron localizarlo; estuvo en algún lugar del edificio del ciclotrón.

\section{LA INSTALACIÓN DEL CICLOTRÓN Y LA PUESTA EN MARCHA}

La instalación del ciclotrón de alguna forma simbolizaba un buen inicio para la naciente Facultad de Ciencias. Se daba una señal al país de que algo interesante estaba ocurriendo en el campo científico. Las autoridades de la universidad consiguieron apoyo nacional e internacional para reunir en este nuevo campus a matemáticos, físicos, biólogos y químicos entusiasmados por desarrollar proyectos avanzados y formar científicos que se suponía que el país necesitaba. Junto al edificio para el ciclotrón se construyeron otros para los departamentos de Física y Matemáticas, un taller mecánico, un taller de electrónica y algunas salas de clases. Para alojar a biólogos y químicos se construyeron varios galpones, en los cuales se habilitaron laboratorios, oficinas, salas de reunión y aulas. Esas construcciones "provisorias" todavía se usan. Ya en 1970 la facultad estaba funcionando casi en plenitud. Hacia la avenida Grecia se construía un edificio de cuatro pisos y un subterráneo para albergar a la biblioteca de la facultad. En su diseño participaron bibliotecarios de la Universidad de California. Este edificio era uno de cuatro semejantes en los cuales se instalarían posteriormente académicos, estudiantes graduados, laboratorios y otros servicios, y por supuesto aquellos que estaban en las construcciones "provisorias". Jóvenes científicos se incorporaron a los departamentos y muchos estudiantes talentosos postularon a las licenciaturas que se ofrecían.

La inauguración del edificio y la puesta en marcha del ciclotrón se realizaron el día 6 de junio de 1967. Se contó con la presencia del Presidente de la República, don Eduardo Frei Montalva, ministros, el Vicerrector de la U. de Chile, Decano Ruy Barbosa, el coordinador general del convenio U. de California- U. de Chile, Decano Ramón Rodríguez, y autoridades universitarias chilenas. La Universidad de California, que donó el acelerador, estuvo representada por el rector de la U. de California Davis, Dr. Emil Mrack, por el director del Crocker Nuclear Laboratory, 
Dr. John Jungerman, y por el ingeniero operador Mr. J. Russel. También asistió el representante de la Ford Foundation, Dr. John Metherton.

A las 11 hrs. el director del Departamento de Física, Dr. Jorge Zamudio Cristi, invitó al Presidente, a autoridades de la Universidad de Chile y a los asistentes especiales a recorrer las instalaciones que se inauguraban. El Dr. Jungerman explicó a los presentes los aspectos técnicos del acelerador en cuyo diseño él participó. Por circuito cerrado de TV se siguió un experimento demostrativo7. Hacia el término del acto académico hubo protestas de estudiantes, que según la prensa provenían del Instituto Pedagógico8.

\section{RECURSOS HUMANOS}

El equipo de investigadores interesados por usar este acelerador se fue formando con algunos que habían estado en el grupo de físicos nucleares de la Escuela de Ingeniería, otros que volvían después de doctorarse y algunos extranjeros provenientes de Argentina, Francia, Gran Bretaña y Brasil. Hacia 1970 se había formado un grupo de unos 10 a 15 investigadores, entre ellos varios estudiantes tesistas9. Este número se redujo a cero hacia 1975.

El ciclotrón se convirtió en un poderoso atractivo para jóvenes científicos, los que fueron aportando sus conocimientos y entusiasmo para explorar las potencialidades técnicas de este acelerador y de acuerdo a ellas desarrollar líneas de trabajo.

\section{PLANES}

Hay que recordar que este ciclotrón se había construido y usado con un fin muy específico en la UC Davis y se desconocían todas sus limitaciones y potencialidades, las que hubo que evaluar una vez instalado en Chile. En principio, el acelerador tenía la capacidad para variar la energía de protones y partículas alfa entre 1.0 y 10 $\mathrm{MeV}$ y hasta $5 \mathrm{MeV}$ en deuterones.

Se planificaron dos áreas de investigación, una basada en el uso directo de haces de partículas cargadas, protones, deuterones y partículas alfa, con los cuales se planeaba medir secciones eficaces diferenciales en algunas reacciones nucleares, y otra en trabajos con neutrones, que se podrían obtener de ciertas reacciones nucleares usando haces de deuterones y con ellos medir secciones eficaces totales en el rango de los $20 \mathrm{MeV}$.

Para los trabajos con partículas cargadas se adquirió una "cámara de scattering" ORTEC y detectores de $\mathrm{Si}(\mathrm{Li})$. No se disponía de un imán deflector de haz, por lo que esta cámara estaba conectada directamente con el haz emergente desde el 
ciclotrón. Para los trabajos con neutrones se adquirió en Europa un blanco muy delgado de $\mathrm{Zr}$ que estaba dopado con tritio. Al interactuar deuterones con tritio se liberan neutrones, que eran los que se pensaba usar en las mediciones. En los trabajos con neutrones se usaría el bunker especialmente construido. En el desarrollo de estos trabajos se hicieron innovaciones metodológicas que se publicaron en la revista Nuclear Instruments and Methods10. También se exploró la posibilidad de usar los haces de partículas cargadas con fines analíticos, en lo que actualmente se llama Ion Beam Analysis. Se adquirió un detector para rayos X a ser usado en el método PIXE (Particle Induced X-ray Emission).

\section{EL ÉXODO}

La actividad universitaria en sus múltiples expresiones no es ajena al acontecer político del medio en que se inserta. El golpe militar ocurrido el 11 de septiembre de 1970 truncó los planes de desarrollo del grupo de físicos nucleares, tanto en lo personal como en su progreso científico. El interés por usar el acelerador decayó ante lo incierto del futuro inmediato. A partir de esa fecha el grupo se fue reduciendo, pues cada uno emigró en busca de tranquilidad, seguridad y posibilidad de desarrollo. El último investigador en partir fue el autor de este artículo, en julio de 1975.

\section{EL REINICIO}

Hacia fines de 1979 el autor regresó a Chile y se reintegró a la Facultad de Ciencias. Afortunadamente, permanecían en la facultad, aunque desarrollando otras tareas, dos técnicos que con su experiencia pudieron volver a poner en operación al ciclotrón. Ellos eran el ingeniero-operador Sr. H.O. Riquelme y el técnico en radiofrecuencia Sr. A. Barbieri. No resultó fácil obtener haces nuevamente; el acelerador llevaba más de cinco años sin uso y se presentaron problemas de vacío, refrigeración y otros que hubo que aprender a solucionar. De los físicos nucleares que emigraron ninguno volvió, por lo que hubo que empezar a formar un grupo. Afortunadamente, la noticia de que el ciclotrón se ponía nuevamente en marcha despertó un enorme entusiasmo en estudiantes de Licenciatura en Física, que ofrecieron desinteresadamente su tiempo y trabajo para realizar diversas tareas, desde limpiar el laboratorio hasta reparar y probar equipos. Se organizaron seminarios y reuniones de trabajo.

Lentamente el grupo fue creciendo en base a algunos instructores y ex alumnos que fueron a cursar sus estudios de doctorado y volvieron, como Claudio Tenreiro y Giancarlo Ramírez. En este período vinieron varios físicos extranjeros en visitas cortas, sabáticas o más extensas. Entre ellos el Profesor J. Jungerman, el Dr. J. L. 
Romero, el Profesor T. A. Cahill, de la UC Davis, y los Profesores María Ester Brandan y Arturo Menchaca de la Universidad Nacional Autónoma de México.

\section{INVESTIGACIÓN}

Las líneas de investigación se fueron definiendo tanto en base a las posibilidades del acelerador para generar haces de energía variable y de alta corriente, como a los recursos humanos y a los fondos disponibles. El ciclotrón presentó serios problemas técnicos cuya solución se hacía más difícil por cuanto en su diseño no se contempló la posibilidad de remover partes de su estructura interior para tareas de mantención y reparación. Además no existían repuestos para el equipo de radio frecuencia, construido en los años cuarenta. Por ejemplo, el equipo de radio frecuencia había sido usado a bordo de un barco de guerra en la segunda guerra mundial. Esas condiciones limitaron seriamente las posibilidades de realizar experimentos complejos. En el caso de trabajos empleando neutrones, solo se pudo realizar una serie de mediciones de secciones eficaces totales en varios elementos debido a que el ciclotrón no pudo generar corrientes de deuterones con intensidad suficiente, del orden de decenas de microamperes. No obstante, los resultados fueron publicados y están incorporados en el banco de datos nucleares del International Atomic Energy Agency, IAEA.

Dadas las severas limitaciones técnicas solo se podía obtener haces de muy baja intensidad, del orden de nanoamperes, eliminando las posibilidades de realizar trabajos en física nuclear básica que requerían haces estables y de alta intensidad. En tales circunstancias, se puso énfasis en usar el haz de protones en aplicaciones analíticas, especialmente el método PIXE (Particle Induced X-ray Emission), que no necesita altas intensidades. Este método no destruye la muestra analizada y puede detectar la presencia de elementos químicos desde muy livianos, como aluminio, hasta muy pesados, como plomo, dependiendo de la concentración. Se hicieron estudios analíticos en aerosoles atmosféricos y en materiales arqueológicos. El carácter interdisciplinario de estos trabajos estimuló la colaboración entre físicos, químicos, ecólogos y arqueólogos de distintas unidades académicas.

\section{EL APORTE DEL CICLOTRÓN A LA FORMACIÓN DE RECURSOS HUMANOS}

El ciclotrón y el laboratorio de física nuclear asociado a él permitieron ofrecer cursos de física nuclear experimental a estudiantes de pre y posgrado. Quince estudiantes hicieron sus trabajos de tesis experimentales usando los haces de iones generados por el ciclotrón 


\section{PRODUCTIVIDAD CIENTÍFICA}

Mientras el ciclotrón estuvo operativo permitió desarrollar investigación básica y aplicada, cuyos resultados se expresan en 21 publicaciones en revistas internacionales, 27 en revistas nacionales con comité editor y 68 presentaciones en reuniones científicas nacionales e internacionales.

\section{EPÍLOGO}

La antigüedad de las piezas usadas en la construcción del ciclotrón y la consiguiente dificultad para conseguir repuestos, junto con un diseño muy compacto que hacía prácticamente imposible acceder a su interior, hizo que las reparaciones fueran cada vez más difíciles y prolongadas. Hacia el año 1994 el ciclotrón presentó demasiados problemas técnicos que resultaron imposibles de solucionar, impidiendo definitivamente sustentar algún trabajo científico. La vida útil de este acelerador de partículas se extendió mucho más de lo que sus constructores pensaron en los años cincuenta.

El grupo de físicos nucleares que se había integrado en su entorno se abocó a la búsqueda de otro acelerador de mejor calidad que permitiera mantener activa esta línea de investigación en la universidad y en el país. Cabe mencionar que ha sido la Universidad de Chile la única institución académica que ha contado con aceleradores de partículas para investigar en física nuclear básica y realizar aplicaciones. Se buscaba un acelerador que apoyara, por ejemplo, estudios de reacciones nucleares de interés astrofísico, aplicaciones analíticas en ciencias de los materiales y en ciencias ambientales, así como que permitiera realizar estudios de daños biológicos producidos con iones. Como es propio de una institución académica, interesaba continuar con el aporte de un laboratorio de física nuclear a la formación de recursos humanos en física experimental. Las aspiraciones del grupo tuvieron éxito cuando en 1996, en concurso internacional, el grupo obtuvo la donación de un acelerador Van de Graaff de parte del laboratorio de Lucent Technologies, New Jersey, EE.UU.

En resumen, la traída a Chile del ciclotrón donado por la UC Davis a la Universidad de Chile permitió:

1. Mantener la actividad científica en física nuclear experimental, iniciada en 1955 con el acelerador Cockcroft-Walton.

2. Contribuir a la formación de físicos nucleares.

3. Promover la investigación interdisciplinaria con aplicaciones de técnicas analíticas nucleares no destructivas. 
A los logros específicos mencionados hay que agregar que independientemente de sus limitaciones técnicas, el ciclotrón se convirtió en un símbolo de progreso científico en los inicios de la Facultad de Ciencias.

\section{NOTAS ANEXAS}

1. Los aceleradores de partículas cargadas se diseñaron y construyeron con el objetivo de explorar el núcleo atómico, tal como lo hizo E. Rutherford en 1909, utilizando partículas alfas emitidas por núcleos radioactivos naturales. Para conocer la estructura del núcleo se necesitaba disponer de otros proyectiles, como protones, deuterones y alfas de variada energía y mayor intensidad que la aportada por fuentes radiactivas. En Inglaterra, en Cambridge, J. Walton y Cockcroft construyeron un acelerador electrostático que funcionó por primera vez en 1931. Los iones ganan energía al desplazarse entre los extremos de un campo eléctrico, por lo que necesitan altos voltajes, en ese caso del orden de cientos de KV. La energía se expresa en unidades $[\mathrm{eV}]$, que es la energía que adquiere un electrón al desplazarse entre dos puntos con diferencia de potencial de 1 Volt. Por otra parte, en los EE.UU., en la Universidad de California, Berkeley, los físicos e ingenieros encabezados por E. Lawrence construyeron un acelerador diferente. En este caso el ión adquiere energía cada vez que cruza una zona con una diferencia de potencial en el interior de un campo magnético. Por efecto de este campo la partícula cargada describe trayectorias curvas cada vez de mayor radio y mayor energía, cíclicamente, de ahí el nombre de ciclotrón. El primer haz se obtuvo en 1932 y de ahí en adelante se construyeron ciclotrones de cada vez mayor energía.

2. Durante la presidencia de John Kennedy se puso en marcha la iniciativa norteamericana llamada Alianza para el Progreso, iniciada en 1961, que promovía la vinculación entre un estado de los EE.UU. y un país de Latinoamérica, con el objetivo de que estos últimos aceleraran su progreso en distintos campos, tales como el académico, industrial y otros. Chile quedó vinculado con el estado de California. Dentro de este contexto se estableció un convenio entre la Universidad de California y la Universidad de Chile, con la finalidad de favorecer la formación de científicos y potenciar la investigación científica que se desarrollaba en nuestra universidad.

3. John Jungerman se graduó en UC Berkeley y participó en la primera prueba de un artefacto nuclear que estalló en el desierto Alamo Gordo en 1945. Como profesor de la U. de California tuvo un destacado rol en el desarrollo del laboratorio de física nuclear en la Davis y en la instalación en ese campus de los cual fue su director. Tuvo un activo rol en el Convenio entre la U. de California 
y la U. de Chile, especialmente en la donación del ciclotrón de 22”. Nos visitó en varias ocasiones y estuvo en una estadía sabática en 1992. Falleció en 2014.

4. Las características técnicas de este ciclotrón se publicaron en la revista Nuclear Instruments and Methods, 18, 19 (1962) 120-124, por J. A. Jungerman, N. F. Peek and C. G. Patten.

5. “Angular Correlation Measurement of ${ }^{12} C\left(n, n^{\prime} \gamma\right)^{12} C$ ". J. Zamudio, J.L. Romero and J.R. Morales. Nucl. Physics A. 96, 449-465 (1967).

6. Neutrones se pueden obtener como resultado de la reacción nuclear en que deuterones interactúan con núcleos de tritio, $3 \mathrm{H}(\mathrm{d}, \mathrm{n}) 4 \mathrm{He}$.

7. Diario El Mercurio. 6 de junio de 1967.

8. Diario La Tercera. 7 de junio de 1967.

9. Nómina de investigadores que participaron en el ciclotrón en distintos períodos:

1967-1975

Jorge Zamudio

Lipo Birstein

Tom W. Conlon

Alberto Filevich

Juan L. Romero

José Roberto Morales

Alex Trier

Patricio Martens

Enrique Espeche

Miguel Roth

C. Suraqui

Alice Rivera

M. Suraqui
1980-1995

José Roberto Morales

Mario Ávila

María Dinator

Carlos Romo

Osvaldo Maldonado

Felipe Llona

Claudio Tenreiro

Ricardo Yáñez

10. "A simple time of flight method to measure the beam energy of a cyclotron". J. L. Romero, H. Massmann, F. P. Brady and J. Zamudio. Nuclear Instruments \& Methods 100 (1972) 551-552.

"A cooling system for thin triated titanium targets". J.R. Morales, J.L. Romero, and M.E. Brandán. Nucl. Instr. \& Methods, 119, 91-95 (1974).

"Two simple circuits for in-beam $\mu$ and ms time measurements". A. Filevich and M. Lagos. Nuclear Instruments \& Methods 122 (1974) 605-609.

"A termal and fast neutron monitor for use in a subcritical facility". L. Birstein, P. Martinez, A. Filevich and F. Li. Nuclear Instruments \& Methods 116 (1974) 615-619. 
11. ciclotrones de 22" y de 56", conformando el Crocker Nuclear Laboratory, del ESTUDIANTES CUYAS TESIS SE REALIZARON EN EL CICLOTRÓN Iván Schuller. Licenciado en Física. U. de Chile. 1970 Herbert Massmann. Licenciado en Física. U. de Chile 1971 Miguel A. Lagos. Licenciado en Física. U. de Chile. 1971 Pedro Menares. Licenciado en Física. U. de Chile. 1973 María E. Brandan. Licenciado en Física. U. de Chile. 1973 Claudio Zanelli. Licenciado en Física. U. de Chile. 1974 José Pedro Donoso. Licenciado en Física. U. de Chile. 1976 Carlos Oyarzún. Licenciado en Física. U. de Chile. 1977 Jaime Valls A. Licenciado en Física. U. de Chile 1982 Joel F. Saavedra. Licenciado en Física. USACH. 1993. Sidney Villagrán. Licenciado en Física. USACH. 1993. Carlos Romo K. Magíster en Física. U. de Chile.1983. Pedro Cerda S. Magíster en Física. U. Austral. 1985. Luis. O. Figueroa. Magíster en Física. U de Chile. 1988. Felipe Llona R. Magíster en Física. U. de Chile. 1991.

\section{PUBLICACIONES CON RESULTADOS OBTENIDOS EN EXPERIMENTOS CON EL CICLOTRÓN EN CHILE}

En el período en que estuvo operando el ciclotrón se publicaron 27 trabajos ( 21 en revistas internacionales y seis en revistas nacionales)

1. "A simple time of flight method to measure the beam energy of a cyclotron". J. L. Romero, H. Massmann, F. P. Brady and J. Zamudio. Nuclear Instruments \& Methods 100 (1972) 551-552.

2. "Two simple circuits for in-beam $\mu$ and ms time measurements". A. Filevich and M. Lagos. Nuclear Instruments \& Methods 122 (1974) 605-609.

3. "A termal and fast neutron monitor for use in a subcritical facility". L. Birstein, P. Martinez, A. Filevich and F. Li. Nuclear Instruments \& Methods 116 (1974) 615-619.

4. "A cooling system for thin triated titanium targets". J.R. Morales, J.L. Romero, and M.E. Brandán. Nucl. Instr. \& Methods, 119, 91-95 (1974).

5. "Determinación de concentraciones de elementos livianos en aerosoles por emisión Xinducida por deuterones”. J. R. Morales y C. Romo. Nucleotécnica vol. 3, No. 5, 45-53 (1983).

6. "Nitrogen content determination using the ${ }^{14} \mathrm{~N}(p, a){ }^{11} C$ ". J.R. Morales, M.I. Dinator, P. Cerda, and J. Junot. J. Radioanal. Nucl. Chem. Letters 96 No.6, 601-610 (1985). 
7. "Determination of I $(K \beta) / I(K a)$ in elements with $21<Z<35$ by deuteron impact". J.R. Morales, J. Valls, R. Elías, and C. Romo. Nucl. Instr. and Methods B, vol. 9, 6-10 (1985).

8. "Estudio de la eficiencia de los filtros Nuclepore en la colección de aerosoles atmosféricos". C. Romo y J. R. Morales. Nucleotécnica 4, No. 8 (1985) 37-40.

9. "Charge effect in Nuclepore filters". C. Romo-Kroeger and J.R. Morales. Physica Scripta vol. 37, 270-273 (1988).

10. "Risks of airborne particulate exposure in a copper mine in Chile". C. Romo-Kroeger,J.R. Morales, F. Llona, P. Auriol and J.E. Wolleter. Industrial Health, vol.27, $\mathrm{N}^{\circ}$ 2 (1989) 95-99.

11. "Measurable Electrostatic Effects in Nuclepore Filters". Carlos M. Romo-Kroger. JAPCA Journal, Vol. 39 N 11, Nov. 1989.

12. "Elemental Analysis of Airborne Particulates in Chile". Carlos M. Romo-Kroeger. Environmental Pollution 68 (1990) 161-170.

13. "Deuteron Excitation as an Alternative in PIXE Analysis". C. M. Romo-Kroeger. Nucl. Instr. \& Meth. In Phys. Research B49 (1990) 29-32.

14. "Time-dependent charge in dielectric membrane filters". Carlos Manuel Romo-Kroeger. Journal of Electrostatic, 25 (1990) 145-154.

15. Protein determination in seeds by proton activation”. J.R. Morales, M.I. Dinator and P. Cerda. Nucl. Instr. and Methods in Phys. Res. B, vol. 40/41, 1196-1198 (1990).

16. "Characterization of colour pigments in pre-Columbian Chilean potteries by PIXE elemental analysis". M.I. Dinator and J.R. Morales. J. of Radioanal. Nucl. Chem. Articles. Vol.140, No.1 (1990) 143-157.

17. "A facility to measure fast neutron cross sections at energies around $20 \mathrm{MeV}$ ". J.R. Morales, L.O. Figueroa, P. Martens and J.L. Romero. Nucl. Inst. and Methods in Phys. Res. A, 300 (1991) 312-320.

18. "Efecto del traslado y embarque de minerales sobre la calidad del aire en una ciudad-puerto de Chile”. L.R. Vallejo, J.W. Fuenteseca, C.M. Romo, J.R. Morales y F. Llona INTERCIENCIA Jul-Ag. 1991, vol.16, No.4, 187-195.

19. "Major Components of Aerosols Emitted by Lonquimay Volcano, Chile". J.R. Morales, M.I. Dinator, F. Llona, and C. Romo-Kroeger. J.of Radioanal. and Nucl. Chem. Articles. Vol. 172, No.1 (1993) 181-192.

20. "Intercomparación de concentraciones elementales en aerosoles atmosféricos determinadas por PIXE y análisis por activación neutrónica”. V. Cassorla, X. Rojas, N. Gras, L. Chuaqui, M.I. Dinator, J.R. Morales, F. Llona y C. Romo-Kroeger. Nucleotécnica Año 13, No. 24 (Junio 1993) 31-41.

21. "Heavy metals in the atmosphere coming from a copper smelter in Chile". C.M. RomoKroeger, J.R. Morales, M.I. Dinator, F. Llona, and L.C. Eaton. Atmospheric Environment Vol. 28 No. 4 pp. 705-711, 1994. 
22. "Sample preparation of archaeological materials for PIXE analysis". J.R. Morales, M.I. Dinator, F. Llona, J. Saavedra and F. Falabella. J.of Radioanal. and Nucl. Chem., Lett. Vol.187, No.1, 79-89 (1994).

23. Primeros resultados de un análisis de composición de elementos con técnicas de rayos- $X$ en arqueología”. F. Falabella, E. Aspillaga, R. Morales, M. I. Dinator, F. Llona. Boletín del Museo Regional de la Araucanía, No. 51.pp 53-58. 1994.

24. Caracterización del material particulado atmosférico en suspensión en Santiago de Chile. Parte VII: Determinación elemental mediante PIXE”. J. R. Morales, M. I. Dinator, C. Romo-Kroeger. Nucleotécnica Año 15, No. 28 (Junio 1995) 47-50.

25. "Risk of Airborne Particulate Exposure in Copper Mines". C. M. Romo-Kroeger Chapter 18. Encyclopedia of Environmental Control Technology Vol.17 High Hazard Pollutants. P. Cheremizanoff (Editor). Gulf Publ. Ca. 1995.

26. "Comparative aerosol studies of the Pacific Rim cities: Santiago, Chile (1987); Mexico (1987-1990), and Los Angeles, U. S. A. (1973 and 1987)'”. T. A. Cahill, J. R. Morales and J. Miranda. Atmospheric Environment Vol. 30, No.5 pp 747-749, 1996

27. "Nuevos antecedentes sobre los sistemas culturales en Chile central sobre la base de análisis de composición de elementos". F. Falabella, E. Aspillaga, J. R. Morales, M.I. Dinator, F. Llona. Revista Chilena de Antropología, No.13. 1995-1996. pp 29-59 